\title{
A countable dense homogeneous space with a dense rigid open subspace
}

by

\author{
Jan van Mill (Amsterdam)
}

\begin{abstract}
We show that there is a Polish space which is countable dense homogeneous but contains a dense open rigid connected subset. This answers several questions of Fitzpatrick and Zhou.
\end{abstract}

1. Introduction. All spaces under discussion are separable and metrizable.

A space $X$ is countable dense homogeneous (abbreviated $\mathrm{CDH}$ ) provided that for all countable dense subsets $D$ and $E$ of $X$ there is a homeomorphism $f$ of $X$ such that $f(D)=E$. Bennett [2] showed that a connected, $\mathrm{CDH}$-space is homogeneous.

Fitzpatrick and Zhou [6] proved that there is a connected, locally connected, CDH, Baire Hausdorff space with a dense, open, connected subspace that is not CDH. See Watson and Simon [16] for a completely regular space with similar properties. Fitzpatrick and Zhou [6] asked whether there is such a space that is metrizable. This question was repeated in [7, Problem 2] and specified in $\left[7\right.$, Problem $\left.2^{\prime}\right]$. The aim of this paper is to answer these questions.

Ungar [15] proved that a continuum other than $\mathbb{S}^{1}$ is $\mathrm{CDH}$ if and only if it is strongly $n$-homogeneous for every $n$. It was stated as a corollary to his main results that every open dense subset of a locally compact $\mathrm{CDH}$-space is itself $\mathrm{CDH}$. As Ungar mentioned in a private conversation, the argument for this corollary is incomplete, and it is unclear whether it is true (see also $[8$, p. 2]). Our example is Polish but not locally compact. So the question whether Ungar's result is true remains open. This seems a rather delicate problem. Kennedy [9] proved that if a continuum is 2-homogeneous, and has

2000 Mathematics Subject Classification: Primary 57N20.

Key words and phrases: countable dense homogeneous spaces, rigid open set, convex set, Hilbert space. 
a nontrivial homeomorphism that is the identity on some nonempty open set, then it is strongly locally homogeneous. Hence every open subspace of such a space is $\mathrm{CDH}$.

A space is rigid if the identity is its only homeomorphism. Based on a construction in [13], we will prove the following:

EXAmple 1.1. There is a convex subspace $S$ of Hilbert space $\ell^{2}$ with dense open convex subspace $T$ such that:

(1) $S$ is CDH (hence $S$ is homogeneous),

(2) $T$ is rigid,

(3) $S \times S \approx T \times T \approx \ell^{2}$.

Observe that (3) implies that both $S$ and $T$ are Polish (among other things).

I am indebted to Tadek Dobrowolski for suggesting a simplification in one of my arguments.

\section{Preliminaries}

(A) Notation. If $X$ and $(Y, \varrho)$ are spaces, then $C(X, Y)$ denotes the collection of all continuous functions from $X$ to $Y$. If $f, g \in C(X, Y)$, then

$$
\widehat{\varrho}(f, g)=\sup \{\varrho(f(x), g(x)): x \in X\} .
$$

If $X$ is compact, then $\widehat{\varrho}$ is a metric and the topology induced by $\widehat{\varrho}$ is separable. See e.g. [12, §1.3] for details.

Let $Q$ denote the Hilbert cube $\prod_{n=1}^{\infty}[-1,1]_{n}$ with its admissible metric

$$
\varrho(x, y)=\sum_{n=1}^{\infty} 2^{-n}\left|x_{n}-y_{n}\right| .
$$

We assume that the reader is familiar with the basic notions in infinitedimensional topology and shape theory, see [12] and [3, 11].

We say that an indexing $\left\{X_{n}: n \in I\right\}$ is faithful provided that $X_{n} \neq X_{m}$ if $n \neq m$. We let $\mathbb{I}$ denote the closed unit interval $[0,1]$.

If $f: X \rightarrow Y$ is a function, then

$$
\Gamma(f)=\{(x, f(x)): x \in X\} \subseteq X \times Y
$$

denotes its graph. The identity function on a set $X$ will be denoted by $1_{X}$.

(B) Complements of $\sigma Z$-sets. Our example will be the complement of a $\sigma Z$-set in $Q$. The following result will be important in the construction.

TheOREM 2.1. Let $B_{1}$ and $B_{2}$ be $\sigma Z$-sets in $Q$ for which there exists a homeomorphism $h: Q \backslash B_{1} \rightarrow Q \backslash B_{2}$. Let $\pi_{i}: \overline{\Gamma(h)} \rightarrow Q$ be the projection maps, $i=1,2$. Then

(a) $\pi_{1}$ and $\pi_{2}$ are monotone surjections such that $\pi_{1}^{-1}\left(B_{1}\right)=\pi_{2}^{-1}\left(B_{2}\right)$, 
(b) if $K_{1}$ and $K_{2}$ are compacta in $B_{1}$ respectively $B_{2}$ such that $\pi_{1}^{-1}\left(K_{1}\right)$ $=\pi_{2}^{-1}\left(K_{2}\right)$, then $\operatorname{Sh}\left(K_{1}\right)=\operatorname{Sh}\left(K_{2}\right)$.

Proof. For (a), see the proof of Lemma 3.6 in Anderson, Curtis and van Mill [1].

For (b) we use the technique in the proof of the easy part of Chapman's Complement Theorem (see Chapman [4, 25.1]). For the convenience of the reader, we provide all details.

Since $B_{1}$ is a $\sigma Z$-set, there is a homotopy $F: Q \times \mathbb{I} \rightarrow Q$ such that $F_{0}=1_{Q}$ and $F(Q \times(0,1]) \subseteq Q \backslash B_{1}$ (since we can think of $B_{1}$ as a subset of the pseudo-boundary of $Q$ this is obvious). Similarly, there exists a homotopy $G: Q \times \mathbb{I} \rightarrow Q$ such that $G_{0}=1_{Q}$ and $G(Q \times(0,1]) \subseteq Q \backslash B_{2}$. For each $n$ define $f_{n}, g_{n}: Q \rightarrow Q$ by

$$
f_{n}=h \circ F_{1 / n}, \quad g_{n}=h^{-1} \circ G_{1 / n} .
$$

We will prove that $\mathbf{f}=\left\{f_{n}, K_{1}, K_{2}\right\}: K_{1} \rightarrow K_{2}$ and $\mathbf{g}=\left\{g_{n}, K_{2}, K_{1}\right\}$ : $K_{2} \rightarrow K_{1}$ are shape maps such that $\mathbf{f} \circ \mathbf{g} \simeq \mathbf{1}_{K_{2}}$ and $\mathbf{g} \circ \mathbf{f} \simeq \mathbf{1}_{K_{1}}$. Therefore, $K_{1}$ and $K_{2}$ have the same shape.

To see that $\mathbf{f}$ is a shape map, let $W$ be an arbitrary open neighborhood of $K_{2}$. Since $\pi_{1}$ and $\pi_{2}$ are closed maps, and $\pi_{1}^{-1}\left(K_{1}\right)=\pi_{2}^{-1}\left(K_{2}\right)$, there is an open neighborhood $V$ of $K_{1}$ such that $\pi_{1}^{-1}(V) \subseteq \pi_{2}^{-1}(W)$. Let $U$ be an open neighborhood of $K_{1}$ in $Q$ such that $\bar{U} \subseteq V$. There clearly exists $\varepsilon>0$ such that

$$
F(\bar{U} \times[0, \varepsilon]) \subseteq V .
$$

Observe that $h\left(U \backslash B_{1}\right) \subseteq h\left(V \backslash B_{1}\right) \subseteq W \backslash B_{2}$. Since for $n \geq 1 / \varepsilon$ we have $F_{1 / n}\left\lceil U \simeq F_{1 /(n+1)}\left\lceil U\right.\right.$ (in $V \backslash B_{1}$ ), it follows that $f_{n}\left\lceil U \simeq f_{n+1}\lceil U\right.$ (in $W$ ). Hence $\mathbf{f}$ is indeed a shape map, and similarly it follows that $\mathbf{g}$ is a shape map.

To prove $\mathbf{g} \circ \mathbf{f} \simeq \mathbf{1}_{K_{1}}$, let $U$ be an arbitrary open neighborhood of $K_{1}$.

Claim 1. There are open neighborhoods $W$ and $N$ of $K_{2}$ and $\varepsilon_{1}>0$ such that

(1) $G\left(\bar{W} \times\left[0, \varepsilon_{1}\right]\right) \subseteq N$

(2) $h^{-1}\left(N \backslash B_{2}\right) \subseteq U$.

Proof. As above, there is an open neighborhood $N$ of $K_{2}$ such that $\pi_{2}^{-1}(N) \subseteq \pi_{1}^{-1}(U)$. Then clearly $h^{-1}\left(N \backslash B_{2}\right) \subseteq U$. Now let $W$ be an open neighborhood of $K_{2}$ such that $\bar{W} \subseteq N$ and find $\varepsilon_{1}>0$ such that $G(\bar{W} \times$ $\left.\left[0, \varepsilon_{1}\right]\right) \subseteq N$.

By exactly the same method, it is clearly possible to prove the following:

Claim 2. There are open neighborhoods $V$ and $M$ of $K_{1}$ and $0<\varepsilon_{2} \leq \varepsilon_{1}$ such that 
(3) $F\left(\bar{V} \times\left[0, \varepsilon_{2}\right]\right) \subseteq M \subseteq U$,

(4) $h\left(M \backslash B_{1}\right) \subseteq W$.

Then for $n \geq 1 / \varepsilon_{2}$ we have $\left(g_{n} \circ f_{n}\right)\left|V=\left(h^{-1} \circ G_{1 / n} \circ h \circ F_{1 / n}\right)\right| V$. Define $S: V \times[0,1 / n] \rightarrow Q$ by

$$
S(x, t)=h^{-1} G_{t} h F_{1 / n} .
$$

Then clearly $S$ is well-defined and continuous, $S_{0}=F_{1 / n} \backslash V$ and $S_{1 / n}=$ $\left(g_{n} \circ f_{n}\right) \nmid V$. If $x \in V$ is arbitrary then, by $(3), S(x, 0)=F_{1 / n}(x) \in M \subseteq U$. Moreover, if $t \in(0,1 / n]$, then by (3) and (4), $h F_{1 / n}(x) \in W \backslash B_{2}$, and so by (1) and (2),

$$
S(x, t)=h^{-1} G_{t} h F_{1 / n}(x) \in h^{-1} G_{t}\left(W \backslash B_{2}\right) \subseteq h^{-1}\left(N \backslash B_{2}\right) \subseteq U .
$$

Hence $S_{0}$ and $S_{1 / n}$ are homotopic in $U$, and the range of $S_{0}$ is contained in $M$. Since by (3) also $F_{1 / n}\lceil V$ and the identity on $V$ are homotopic in $U$, this shows that the identity on $V$ and the function $\left(g_{n} \circ f_{n}\right) \mid V$ are homotopic (in $U$ ). So we conclude that $\mathbf{g} \circ \mathbf{f} \simeq \mathbf{1}_{K_{1}}$. It follows similarly that $\mathbf{f} \circ \mathbf{g} \simeq \mathbf{1}_{K_{2}}$.

3. Dense collections of compacta. Let $X$ be a nonempty compact space. We say that a countable collection of $Z$-sets $X$ in $Q$ is $X$-dense if

(1) $X$ is pairwise disjoint and every $X^{\prime} \in X$ is homeomorphic to $X$,

(2) for every $f \in C(X, Q)$ and $\varepsilon>0$ there are an $X^{\prime} \in \mathcal{X}$ and a homeomorphism $\alpha: X \rightarrow X^{\prime}$ such that $\widehat{\varrho}(\alpha, f)<\varepsilon$.

The basic properties of $X$-dense collections that are important to us are listed in the following result.

Proposition 3.1 ([13]). Let $X$ be a nonempty compact space.

(a) There is an $X$-dense collection of $Z$-sets in $Q$.

(b) Let $\mathcal{S}$ and $\mathcal{T}$ be $X$-dense collections of $Z$-sets in $Q$. Then there is an arbitrarily close to the identity homeomorphism $h: Q \rightarrow Q$ such that $h(\bigcup \mathcal{S})=\bigcup \mathcal{T}$.

Now let $Q$ be a $Q$-dense collection of $Z$-sets in $Q$ (Proposition 3.1(a)), and put $Y=Q \backslash \bigcup \mathcal{Q}$. Then $Y$ is a dense $G_{\delta}$-subset of $Q$ and hence is Polish. It was shown in [13] that $Y$ is an example of a $\mathrm{CDH}$-space which is not strongly locally homogeneous. We will show here that $Y$ has many other interesting properties. In fact, $Y$ is homeomorphic to the space $S$ in Example 1.1.

Let $\left\{Q_{n}: n \in \mathbb{N}\right\}$ be a faithful enumeration of $Q$. Since there are arbitrarily small maps $Q \rightarrow Q \backslash Y$, it follows that every compact subspace of $Y$ is a $Z$-set in $Q$. 
Lemma 3.2. There are a compact set $K$ in $Q \backslash Y$ and an open base $\mathcal{B}$ for $Q \backslash K$ such that

(1) for every $B \in \mathcal{B}, \bar{B} \cap K=\emptyset$,

(2) for all $B, B^{\prime} \in \mathcal{B}$ such that $\bar{B} \cap \overline{B^{\prime}}=\emptyset$, there exists $n$ such that $Q_{n} \cap K \neq \emptyset, Q_{n} \cap B \neq \emptyset$ but $Q_{n} \cap B^{\prime}=\emptyset$.

Proof. Let $K^{\prime}$ denote an arbitrary convergent sequence with limit in $Y$. In addition, let $\mathcal{A}$ be a countable open base for $Q \backslash K$ such that for every $A \in \mathcal{A}$,

(3) $\bar{A} \cap K=\emptyset$,

(4) $Q \backslash \bar{A}$ is connected.

Let $\left\{\left(A_{n}, A_{n}^{\prime}\right): n \in \mathbb{N}\right\}$ enumerate all pairs $\left(A, A^{\prime}\right)$ of elements of $\mathcal{A}$ such that $\bar{A} \cap \overline{A^{\prime}}=\emptyset$.

Enumerate $K^{\prime}$ faithfully as $\left\{e_{n}: n \in \mathbb{N}\right\}$. Let $D=\left\{d_{n}: n \in \mathbb{N}\right\}$ be a faithfully indexed subset of $Y$ such that $d_{n} \in A_{n}$ for every $n$. We will construct for every $n$ an imbedding $g_{n}: Q \rightarrow Y$ such that

(5) $d_{n}, e_{n} \in g_{n}(Q)$

(6) $g_{n}(Q) \cap\left(\left(D \backslash\left\{d_{n}\right\}\right) \cup \bigcup_{i=1}^{n-1} g_{i}(Q) \cup\left(K^{\prime} \backslash\left\{e_{n}\right\}\right)\right)=\emptyset$,

(7) $g_{n}(Q) \cap \overline{A_{n}^{\prime}}=\emptyset$.

The inductive construction is a triviality. Put $M=Q \backslash \overline{A_{n}^{\prime}}$. Then $M$ is a connected open subset of $Q$ containing both $d_{n}$ and $e_{n}$. Let $J$ be a path in $M$ connecting $d_{n}$ and $e_{n}$, and let $\xi: Q \rightarrow J$ be any continuous surjection. Observe that

$$
T=\bigcup \mathcal{Q} \cup\left(D \backslash\left\{d_{n}\right\}\right) \cup \bigcup_{i=1}^{n-1} g_{i}(Q) \cup\left(K^{\prime} \backslash\left\{e_{n}\right\}\right)
$$

belongs to $z_{\sigma}(Q)$ and misses $\left\{d_{n}, e_{n}\right\}$. Hence $M \cap T$ belongs to $z_{\sigma}(M)$. So by $[12,7.3 .5(\mathrm{~g})], \xi: Q \rightarrow J$ can be approximated by an imbedding $g_{n}: Q \rightarrow$ $Y \cap M$ satisfying $(5)_{n}$ and $(6)_{n}$. And it is trivial that $(7)_{n}$ holds.

Now observe that the sequence

$$
\left\{Q_{n}: n \in \mathbb{N}\right\} \cup\left\{g_{n}(Q): n \in \mathbb{N}\right\}
$$

is $Q$-dense because the sequence $\left\{Q_{n}: n \in \mathbb{N}\right\}$ is. Consequently, by Proposition 3.1(b) there is a homeomorphism $\alpha: Q \rightarrow Q$ such that

$$
\alpha\left(\bigcup_{n=1}^{\infty} Q_{n} \cup \bigcup_{n=1}^{\infty} g_{n}(Q)\right)=\bigcup_{n=1}^{\infty} Q_{n} .
$$

It is clear that $K=\alpha\left(K^{\prime}\right)$ and $\mathcal{B}=\{\alpha(A): A \in \mathcal{A}\}$ are as required.

Let $Z=\left\{y_{n}: n \in \mathbb{N}\right\}$ be a discrete subset of $Y$ such that $\bar{Z} \backslash Z=K$. Since $Y$ is dense and $K$ is nowhere dense in $Q$, it is clearly possible to pick such a sequence. Since $Q \backslash Y \in z_{\sigma}(Q)$, the constant function $\mathbb{S}^{n} \rightarrow\left\{y_{n}\right\}$ can 
be approximated arbitrarily closely by an imbedding $\mathbb{S}^{n} \rightarrow Y([12,7.3 .5(\mathrm{~g})])$ (here $\mathbb{S}^{n}$ denotes the $n$-sphere of course). This means that we can enlarge the singleton set $\left\{y_{n}\right\}$ to a copy of the $n$-sphere $\mathbb{S}_{n}$ in $Y$ such that

(A) if $n \neq m$, then $\mathbb{S}_{n} \cap \mathbb{S}_{m}=\emptyset$,

(B) $\lim _{n \rightarrow \infty} \operatorname{diam}\left(\mathbb{S}_{n}\right)=0$.

Observe that (B) implies (C), where

(C) $\bar{S} \backslash S=K$, and $S=\bigcup_{n=1}^{\infty} \mathbb{S}_{n}$.

There is nothing special about the use of spheres in our construction. From the remaining part of this paper it will be clear that any sequence consisting of compacta of pairwise different but nontrivial shape will do equally well.

4. The example. Consider the following collection of pairwise disjoint continua in $Q$ :

$$
\mathcal{P}=\left\{Q_{n}: n \in \mathbb{N}\right\} \cup\left\{\mathbb{S}_{n}: n \in \mathbb{N}\right\} .
$$

Let $Y=Q \backslash \bigcup_{n=1}^{\infty} Q_{n}$ be the space introduced in $\S 3$, and put $X=Y \backslash$ $\bigcup_{n=1}^{\infty} \mathbb{S}_{n}$. Then $X$ is dense in $Q$, being the complement of a $\sigma Z$-set in $Q$, and is a dense open subset of $Y$.

Theorem 4.1. $Y$ is $\mathrm{CDH}$ and $X$ is rigid.

Proof. That $Y$ is $\mathrm{CDH}$ was proved in [13]. To prove that $X$ is rigid, let $h$ be any homeomorphism of $X$. Consider the graph $\Gamma(h)$ of $h$ in $Q \times Q$. We adopt the notation of Theorem 2.1 (with $B_{1}=B_{2}=Z$ ).

We will first prove by the method of $[13,4.2]$ that $h$ permutes $\mathcal{P}$.

Claim 1. There is a bijection $\alpha: \mathcal{P} \rightarrow \mathcal{P}$ such that for every $A \subseteq X$ and $P \in \mathcal{P}$, if $\bar{A} \cap P \neq \emptyset$ then $\overline{h(A)} \cap \alpha(P) \neq \emptyset$ (here closure means closure in $Q)$.

Proof. Take an arbitrary $P \in \mathcal{P}$. Then $\pi_{1}^{-1}(P)$ is a continuum since $\pi_{1}$ is monotone (Theorem 2.1(a)). Hence $\pi_{2}\left(\pi_{1}^{-1}(P)\right)$ is a continuum, and is therefore, by the Sierpiński theorem from [14], contained in some member $P^{\prime} \in \mathcal{P}^{\prime}$. Since $\pi_{2}$ is monotone as well, $\pi_{2}^{-1}\left(P^{\prime}\right)$ is a subcontinuum of $\overline{\Gamma(h)}$ containing $\pi_{1}^{-1}(P)$. Hence $\pi_{1}\left(\pi_{2}^{-1}\left(P^{\prime}\right)\right)$ is a subcontinuum of $Z$ containing $P$. But again by the Sierpiński theorem, $P$ is a maximal subcontinuum of $\bigcup \mathcal{P}$, hence $\pi_{1}\left(\pi_{2}^{-1}\left(P^{\prime}\right)\right)=P$. From this we conclude that $\pi_{1}^{-1}(P)=\pi_{2}^{-1}\left(P^{\prime}\right)$. Define $\alpha(P)=P^{\prime}$. It is clear that $\alpha$ is a bijection.

Let $A \subseteq X$ and $P \in \mathcal{P}$ be such that $\bar{A} \cap P \neq \emptyset$. Pick an arbitrary open neighborhood $U$ of $\alpha(P)$. Then $\pi_{2}^{-1}(U)$ is an open neighborhood of $\pi_{2}^{-1}(\alpha(P))=\pi_{1}^{-1}(P)$. Since $\pi_{1}$ is a closed map, there is an open neighborhood $V$ of $P$ such that $\pi_{1}^{-1}(V) \subseteq \pi_{2}^{-1}(U)$. Pick an element $a \in A \cap V$. Then $(a, h(a)) \in \pi_{1}^{-1}(V)$, hence $h(a)=\pi_{2}(a, h(a)) \in \pi_{2}\left(\pi_{1}^{-1}(V)\right) \subseteq U$, as required. 
Claim 2. For every $n \in \mathbb{N}, \alpha\left(\mathbb{S}_{n}\right)=\mathbb{S}_{n}$. Moreover, if $Q_{n} \cap K \neq \emptyset$, then $\alpha\left(Q_{n}\right)=Q_{n}$.

Proof. That $\alpha\left(\mathbb{S}_{n}\right)=\mathbb{S}_{n}$ for every $n$ is a direct consequence of Theorem 2.1(b). Simply observe that $Q$ has trivial shape, and $0 \neq \operatorname{Sh}\left(\mathbb{S}_{n}\right) \neq$ $\operatorname{Sh}\left(\mathbb{S}_{m}\right) \neq 0$ if $n \neq m$.

Assume that $Q_{n} \cap K \neq \emptyset$ for some $n$, and let $p \in K \cap Q_{n}$. There is a sequence $\left(n_{i}\right)_{i}$ such that the sequence $\left(\mathbb{S}_{n_{i}}\right)_{i}$ of continua converges to $p$. By compactness it is clear that $\pi_{1}^{-1}(p) \cap \overline{\bigcup_{i=1}^{\infty} \pi_{1}^{-1}\left(\mathbb{S}_{n_{i}}\right)} \neq \emptyset$. Hence $\pi_{2}\left(\pi_{1}^{-1}(p)\right) \cap$ $\left.\overline{\bigcup_{i=1}^{\infty} \pi_{2}\left(\pi_{1}^{-1}\left(\mathbb{S}_{n_{i}}\right)\right.}\right) \neq \emptyset$, and therefore since $\alpha\left(\mathbb{S}_{j}\right)=\mathbb{S}_{j}$ for every $j, \alpha\left(Q_{n}\right) \cap$

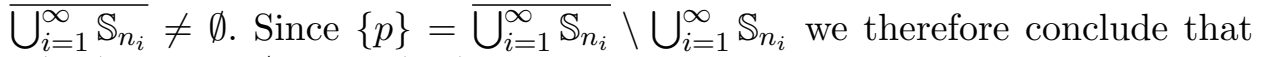
$\alpha\left(Q_{n}\right) \cap Q_{n} \neq \emptyset$, i.e., $\alpha\left(Q_{n}\right)=Q_{n}$.

Striving for a contradiction, assume that there exists $x \in X$ such that $h(x) \neq x$. Since $\{x, h(x)\} \cap K=\emptyset$, there are $B, B^{\prime} \in \mathcal{B}$ such that $x \in B$, $h(B \cap X) \subseteq B^{\prime} \cap X$, and $\bar{B} \cap \overline{B^{\prime}}=\emptyset$. By Lemma 3.2 there exists $n$ such that $Q_{n} \cap K \neq \emptyset, Q_{n} \cap B \neq \emptyset$ but $Q_{n} \cap \overline{B^{\prime}}=\emptyset$. Pick a sequence $\left(x_{i}\right)_{i}$ in $B \cap X$ that converges to an element of $Q_{n} \cap \bar{B}$. By Claim 1, the sequence $\left(h\left(x_{i}\right)\right)_{i}$ has a cluster point in $\alpha\left(Q_{n}\right)$. But $\alpha\left(Q_{n}\right)=Q_{n}$ by Claim 2 and all cluster points of $\left(h\left(x_{i}\right)\right)_{i}$ are contained in $\overline{B^{\prime}}$, which is disjoint from $Q_{n}$. This is a contradiction.

We will now show that $Y$ has the additional properties promised in Example 1.1. That $(Y, X)$ is homeomorphic to a pair of convex subsets of $\ell^{2}$ follows from [1, Theorem 3.1] since $Q \backslash X$ is a $\sigma Z$-set in $Q$. By observing that the identity function $Q \rightarrow Q$ can be approximated arbitrarily closely by maps $Q \rightarrow Q \backslash X, Y \times Y \approx X \times X \approx \ell^{2}$ follows from [1, Theorem 3.5].

REMARK 4.2. In [13, Remark 4.5], it was shown that if $h: Y \rightarrow Y$ is any homeomorphism that restricts to the identity on a certain zero-dimensional closed subset $L$ of $Y$, then $h$ is the identity. The set $L$ is not a $Z$-set in $Y$, and this is precisely why the argument there worked. With the method used in the proof of Theorem 4.1, it is easy to show that if $h$ is any homeomorphism of $Y$ that restricts to the identity on the countable closed discrete subset $Z$ of $Y$, then $h$ is the identity. Observe that $Z$ is a $Z$-set in $Y$.

REMARK 4.3. We see that $X$ is an example of a rigid space whose square is $\ell^{2}$. Such spaces were earlier constructed by Dijkstra [5]. For a zerodimensional rigid space whose square is homogeneous, see Lawrence [10].

\section{References}

[1] R. D. Anderson, D. W. Curtis and J. van Mill, A fake topological Hilbert space, Trans. Amer. Math. Soc. 272 (1982), 311-321.

[2] R. Bennett, Countable dense homogeneous spaces, Fund. Math. 74 (1972), 189-194. 
[3] K. Borsuk, Theory of Shape, Monograf. Mat. 59, PWN-Polish Sci. Publ., Warszawa, 1975.

[4] T. A. Chapman, Lectures on Hilbert Cube Manifolds, CBMS Reg. Conf. Ser. Math. 28, Amer. Math. Soc., Providence, RI, 1976.

[5] J. J. Dijkstra, A rigid space whose square is the Hilbert space, Proc. Amer. Math. Soc. 93 (1985), 118-120.

[6] B. Fitzpatrick, Jr. and H. X. Zhou, Densely homogeneous spaces. II, Houston J. Math. 14 (1988), 57-68.

[7] - , - Some open problems in densely homogeneous spaces, in: Open Problems in Topology, J. van Mill and G. M. Reed (eds.), North-Holland, Amsterdam, 1990, 251-259.

[8] -, - Countable dense homogeneity and the Baire property, Topology Appl. 43 (1992), 1-14.

[9] J. Kennedy, A condition under which 2-homogeneity and representability are the same in continua, Fund. Math. 121 (1984), 89-98.

[10] L. B. Lawrence, A rigid subspace of the real line whose square is a homogeneous subspace of the plane, Trans. Amer. Math. Soc. 357 (2005), 2535-2556.

[11] S. Mardešić and J. Segal, Shape Theory, North-Holland, Amsterdam, 1982.

[12] J. van Mill, Infinite-Dimensional Topology: Prerequisites and Introduction, NorthHolland, Amsterdam, 1989.

[13] - On countable dense and strong local homogeneity, Bull. Polish Acad. Sci. Math. 53 (2005), 401-408.

[14] W. Sierpiński, Un théorème sur les continus, Tôhoku Math. J. 13 (1918), 300-303.

[15] G. S. Ungar, Countable dense homogeneity and n-homogeneity, Fund. Math. 99 (1978), 155-160.

[16] S. Watson and P. Simon, Open subspaces of countable dense homogeneous spaces, ibid. 141 (1992), 101-108.

Department of Mathematics

Faculty of Sciences

VU University Amsterdam

De Boelelaan 1081a

1081 HV Amsterdam, The Netherlands

E-mail: vanmill@cs.vu.nl 